Abanico Agroforestal. Enero-Diciembre 2020; 2:1-9. http://dx.doi.org/10.37114/abaagrof/2020.8

Artículo Original. Recibido: 16/04/2020. Aceptado: 15/07/2020. Publicado: 25/07/2020.

\title{
Estudio geográfico e identificación de plantas con potencial apícola en Nayarit, México
}

Geographical study and identification of plants with apicultural potential in Nayarit, Mexico

\section{Maricela Martínez-Virgen ${ }^{1 \mathrm{ID}}$, Ricardo Ulloa-Castañeda ${ }^{1 \mathrm{ID}}$, Socorro Salgado- Moreno $^{* * 1} \mathrm{ID}$, Carlos Carmona-Gasca ${ }^{1 \mathrm{ID}}$, Guadalupe Orozco-Benítez ${ }^{1 \mathrm{ID}}$, Sergio Martínez-González*1 ID}

1Universidad Autónoma de Nayarit, Unidad Académica de Medicina Veterinaria y Zootecnia. Nayarit, México. *Autor de correspondencia. ${ }^{* *}$ Autor responsable. Universidad Autónoma de Nayarit, Unidad Académica de Medicina Veterinaria y Zootecnia. Km 3.5 Carretera Compostela-Chapalilla. Compostela, Nayarit, México. CP. 63700. maricela_m@hotmail.com, ulloacar@hotmail.com, coco_salgado@hotmail.com, carmonagasca@gmail.com, mgorozco63@gmail.com, sergio.martinez@uan.edu.mx

\section{RESUMEN}

México es de los principales países productores y exportadores de miel a nivel mundial, esta producción depende de la cantidad y diversidad botánica encontrada regionalmente, de ella dependen las características de la miel, su precio y demanda, de ahí la importancia que los apicultores y autoridades tengan conocimiento, la distribución geográfica y épocas de floración de las plantas con potencial apícola. Por ello, el objetivo de este estudio fue localizar e identificar las plantas con potencial apícola en el estado de Nayarit. Con este fin, se realizaron encuestas a los apicultores del estado de Nayarit, para recopilar información sobre las plantas que las abejas visitan y los nombres comunes de cada una de ellas. Después se acudió a cada sitio donde estaban los apiarios y se realizó la observación directa de las flores, tomando registro fotográfico y ubicación georeferenciada para recolectar muestras de las plantas mencionadas para su futura clasificación e identificación. Se recolectaron 1274 muestras de plantas, y de estas se identificaron 82 especies diferentes de las que se cuenta con nombre científico, nombre común, fotografía y ubicación geográfica, además de otras 95 especies con solo nombre común y científico. El trabajo concluido resulta en el primer censo de especies cuya floración es de importancia en la producción de miel de abeja en la región nayarita.

Palabras clave: abejas, flores, identificación y miel.

\begin{abstract}
Mexico is one of the main honey producing and exporting countries worldwide, this production depends on the quantity and botanical diversity found regionally, the characteristics of honey, its price and demand depend on it, hence the importance that beekeepers and authorities be aware of the geographical distribution and flowering times of plants with apicultural potential. This study aims to locate and to identify the plants with apicultural potential in the state of Nayarit. Given to this, a group of experienced beekeepers were surveyed. The producers reported the type of flowers that bees regularly visit and their common local names for each plant. Every site and apiary were visited, locating them with geolocator and direct observations were made. Additionally, photographs of the flowers were taken, and samples of plants were collected for their identification. In the present study, 1,274 plant-samples were collected, and 82 different species were identified with the corresponding scientific name, common name, photograph, and geographic location, as well as 95 species were also identified with only common and scientific names. The work completed represents the first census of species whose flowering is of importance in the production of honey in the Nayarit region.
\end{abstract}

Keywords: bees, flowers, identification, honey. 


\section{INTRODUCCIÓN}

En el mercado internacional de la miel, los principales países productores son: China, Estados Unidos de Norteamérica, México, Rusia, Argentina, Canadá, Alemania y Japón, con el $40 \%$, pero solo la oferta al mercado mundial es proporcionada por China, Argentina y México, con un $75 \%$ de las exportaciones totales (García-Gómez y Meza-Ramos, 2013).

México es de los principales países productores de miel y exportador a nivel mundial, en ello radica la importancia de estudiar las plantas melíferas. Para mejorar la producción, es necesario que los apicultores y autoridades tengan conocimiento de las plantas con potencial apícola, la distribución geográfica y épocas de floración. En varias regiones del país se han realizado estudios para su ubicación y tipo de plantas (Araujo-Mondragón y Redonda-Martínez, 2019).

Se ha estudiado la flora melífera de distintas regiones, entre ellas: la Sierra de Mazatlán, Jalisco, Colima, Chiapas, Yucatán, el sur del Valle de México, Campeche, Zacatecas, Tabasco, el Valle de Mexicali y Baja California, entre otros. Los estados de Campeche, Chiapas y Yucatán están entre los principales productores de miel del país (AlanizGutiérrez et al., 2017; Araujo-Mondragón y Redonda-Martínez, 2019); por lo que es importante tener esta información en Nayarit.

La composición de los recursos de néctar y polen varían de acuerdo a la distribución de los diferentes climas y flora. En México, estas diferencias permiten definir zonas definidas con diferentes grados de desarrollo y variedad de mieles, en cuanto a sus características de humedad, color, aroma y sabor. Por lo tanto, el país se divide en cinco regiones apícolas: altiplano, golfo, costa del pacífico, norte y península de Yucatán; las cuales son: A) Región Norte: comprendida por las entidades de Baja California, Baja California Sur, Sonora, Chihuahua, Durango, Zacatecas, Coahuila, Nuevo León y parte del norte de Tamaulipas y altiplano de San Luis Potosí; que tiene como característica: la producción de excelente miel, principalmente de mezquite, que es una miel extra clara color ámbar; B) Región de la Costa del Pacífico: formada por los estados de Sinaloa, Nayarit, poniente de Jalisco y Michoacán, Colima, parte de Guerrero, Oaxaca y Chiapas; sus características son: mieles de origen multifloral y de mangle, siendo principalmente obscuras; C) Región del Golfo: que comprende a Veracruz, parte de los estados de Tabasco, Tamaulipas y la Región Huasteca de San Luis Potosí, Hidalgo y Querétaro; con las características: derivadas de miel de cítricos, miel ámbar clara producida principalmente a partir de la flor del naranjo, siendo muy apreciada internacionalmente; D) Región del Altiplano: integrado por las entidades de Tlaxcala, Puebla, México, Morelos, Distrito Federal, Guanajuato, Aguascalientes, la parte oriente de los estados de Jalisco, Michoacán, Guerrero, Oaxaca y Chiapas; así como la parte poniente de Hidalgo y Querétaro, de igual manera la región media de San Luis Potosí, que tiene la característica de tener miel ámbar y miel clara (tipo mantequilla), que tiene demanda en el mercado europeo; E) Región Sureste o Península de Yucatán: formada por Campeche, Yucatán y Quintana Roo y parte de los estados de Chiapas (Noreste) y 
Tabasco (Oriente). La miel que se cosecha es reconocida a nivel internacional, siendo la más importante por su volumen de producción y donde se encuentra la mayor parte de los apicultores del país (SAGARPA, 2018; García-Gómez y Meza-Ramos, 2013).

Nayarit es rico en plantas melíferas y poliníferas, y esto lo aprovechan los apicultores en la producción de miel, generando economía para sus familias. Aunado a que una gran cantidad de cultivos requieren de la presencia de abejas para su polinización y mejora de la producción, más la gran cantidad de manglares, bosques y selvas que dependen de este insecto para su conservación (Ulloa et al., 2014).

El éxito de la apicultura depende de las plantas que se encuentran alrededor de los apiarios, aproximadamente con un radio aproximado de $10 \mathrm{~km}$, por tal razón el apicultor debe acumular experiencia y conocimientos fenológicos, como las fechas de floración, ubicación, vientos, exceso de lluvias, fuentes de agua, incendios; además de los insecticidas, fungicidas, acaricidas y herbicidas que causan la muerte de polinizadores 0 contaminan la miel (SAGARPA, 2018; Vargas et al., 2020).

Datos sobre el impacto de las actividades humanas sobre la flora, revelan que el $36.5 \%$ de estas plantas son afectadas por cambio de uso del suelo, $35.5 \%$ se consideran malezas, $7.5 \%$ sufrieron daños por plagas y también son catalogadas como malezas y $4.3 \%$ son huéspedes de plantas parásitas. Solo el $16.2 \%$ no tiene riesgo alguno, ya que son árboles frutales o especies cultivadas (Hanan y Heike, 2015; Araujo-Mondragón y Redonda-Martínez, 2019). Esta información demuestra la importancia de conservarlas, protegerlas, incluso la diseminación; lo que resultará un mejor ambiente.

El objetivo de este estudio fue localizar e identificar las plantas con potencial apícola en el estado de Nayarit.

\section{MATERIAL Y MÉTODOS}

El estado de Nayarit se encuentra localizado entre las coordenadas geográficas $20^{\circ}$ y $23^{\circ}$ de Latitud Norte y $103^{\circ}$ y $106^{\circ}$ de Longitud Oeste, donde predomina el clima cálido sub húmedo, con lluvias en verano; temperatura promedio anual de $21.3^{\circ} \mathrm{C}$, precipitación promedio de $1152.3 \mathrm{~mm}$ y altitud de $915 \mathrm{~m}$ sobre el nivel del mar. Está dividido en 20 municipios que conforman seis regiones. La primera región denominada Norte, está integrada por los municipios de Acaponeta, Rosamorada, Ruiz, y Tuxpan; la segunda región llamada Centro, está integrada por los municipios de Tepic y Xalisco; la tercera región llamada Sur por su ubicación, comprende los municipios de Ahuacatlán, Amatlán de Cañas, Ixtlán del Río, Jala, San Pedro Lagunillas y Santa María del Oro; la cuarta región conocida como Costa Sur, agrupa a Bahía de Banderas y Compostela; la quinta región denominada Sierra, está integrada por Huajicori, El Nayar y La Yesca; y finalmente la región Costa Norte, integrada por San Blas, Santiago Ixcuintla y Tecuala (García-Gómez y Meza-Ramos, 2013). 
Se realizó encuesta a la mayoría de los apicultores asociados en el Sistema Producto Apícola de Nayarit, donde ellos con su experiencia informaron sobre las flores que las abejas visitan y los nombres comunes de cada planta. Después se acudió a los sitios donde son asentados los apiarios, ubicándolos con geolocalizador y se realizaron observaciones directas, fotografías de las flores y recolección de muestras de las plantas mencionadas, para su futura clasificación e identificación de acuerdo a la morfología y uso de las claves taxonómicas en la Unidad Académica de Agricultura de la Universidad Autónoma de Nayarit, donde se llevó a cabo la identificación de las plantas.

\section{RESULTADOS Y DISCUSIÓN}

Se recolectaron 1274 muestras de plantas, y de éstas se identificaron 82 especies diferentes, de las que se cuenta con nombre científico, nombre común, fotografía y su localización en mapas independientes, como se observa una planta en la figura 1. Ver Información Suplementaria para las 82 especies.

Figura 1. Acacia pennatula (Sclecht. y Cham.) Benth. Nombre común: tepame.

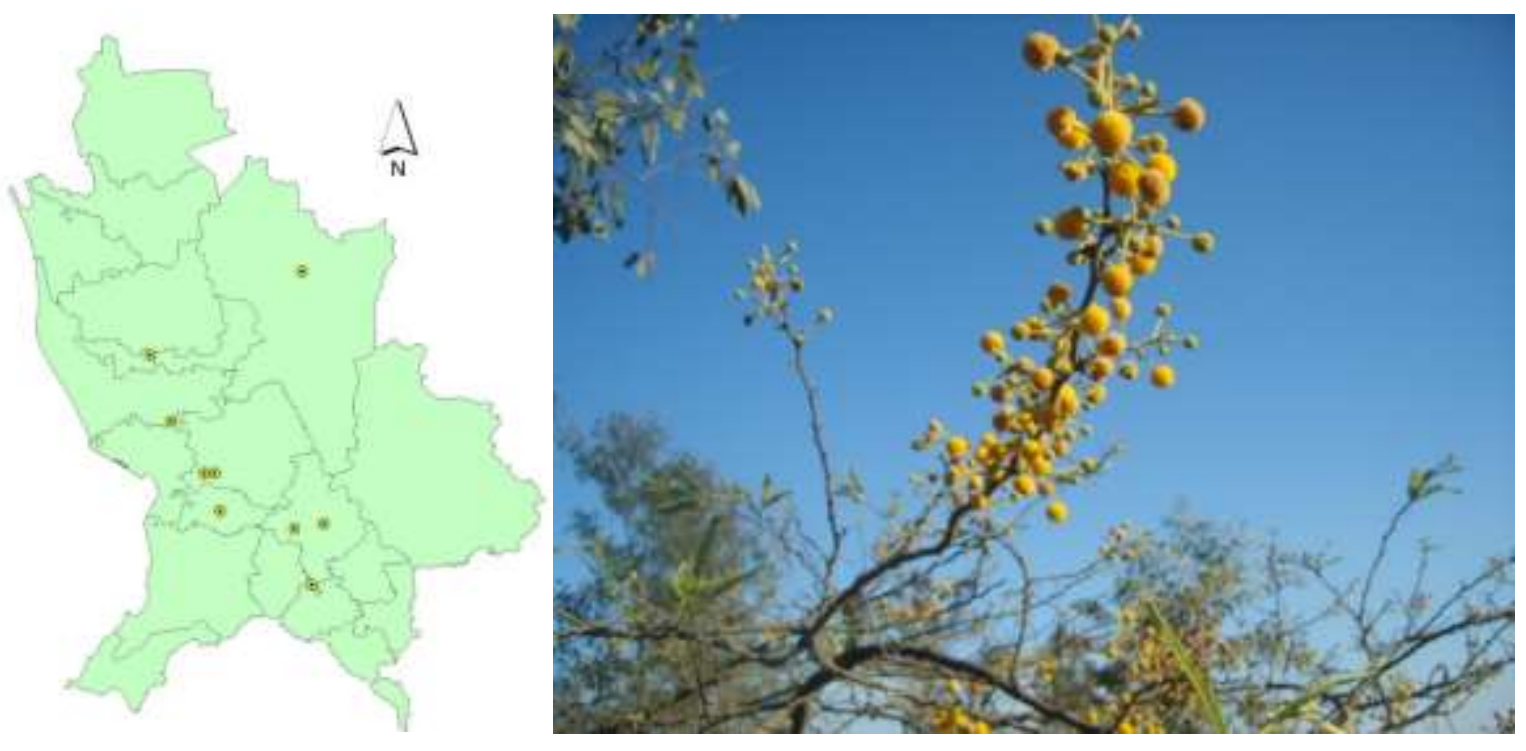

A continuación se presenta un listado de especies de plantas, su nombre común, nombre científico, número de lugares y porcentaje del total de lugares donde fueron encontradas en Nayarit: guajillo (Acacia angustissima (Mill.) Kuntze) con 21 (2.77\%), tepame (Acacia pennatula (Schltdl. y Cham.) Benth) con 9 (1.19\%), capiro (Albizia lebbeck (L.) Benth.) con 2 (0.26\%), cacanacuaste (Apoplanesia paniculata C. Presl) con $3(0.40 \%)$, veintiunilla o venenillo o yerba del sapo (Asclepias curassavica L.) con $3(0.40 \%)$, rosa de mayo o san miguel o san miguelito (Antigonon leptopus Hook. y Arn.) con 5 (0.66\%), gigantillo o zarcillo (Aeschynomene americana L.) con 8 (1.06\%), oreja de mula (Asclepias glaucescens Kunth) con 6 (0.79\%), mangle negro (Avicennia germinans (L.) L.) con 19 (2.51\%), achiote (Bixa orellana L.) con 10 (1.32\%), chicalote de árbol o palo santo (Bocconia arborea S. Watson) con 11 (1.45\%), capomo o ramón (Brosimum 
alicastrum Sw.) con 9 (1.19\%), copalillo o corta pico (Bursera penicillata (DC.) Engl.) con 35 (4.62\%), jiote (Bursera simaruba (L.) Sarg.) con 9 (1.19\%), nanchi (Byrsonima crassifolia (L.) Kunth) con 18 (2.37\%), tacote amarillo o manita de león (Calea urticifolia (Mill.) DC.) con 20 (2.64\%), chivato (Calliandra houstoniana (MII.) Standl.) con 10 (1.32\%), obatel (Casearia nitida (L.) Jacq.) con $5(0.66 \%)$, hierba de la golondrina (Chamaesyce hirta (L.) Millsp.) con $3(0.40 \%)$, barbas de viejo o rabo de iguana (Chamissoa altissima (Jacq.) Kunth) con $3(0.40 \%)$, barbas de chivo (Clematis dioica L.) con $5(0.66 \%)$, palo de rosa amarilla (Cochlospermum vitifolium (Willd.) Spreng.) con 8 (1.06\%), botoncillo (Conocarpus erectus L.) con 20 (2.64\%), mirasol amarillo (Cosmos sulphureus Cav.) con 9 (1.19\%), cuastecomate (Crescentia alata Kunth) con 4 (0.53\%), toloache o floripondio (Datura stramonium L.) con $5(0.66 \%)$, guairaje (Eugenia axillaris (Sw.) Willd.) con $3(0.40 \%)$, jarilla, chía real (Galinsoga parviflora Cav.) con 7 (0.92\%), guásima (Guazuma ulmifolia Lam.) con 28 (3.69\%), hincha huevos (Hippomane mancinella L.) con $5(0.66 \%)$, cardo o viuda (Hydrolea spinosa L.) con $7(0.92 \%)$, guapinol (Hymenaea courbaril L.) con 9 (1.19\%), salvia o salvia real (Hyptis albida Kunth) con 21 (2.77\%), Hyptis suaveolens (L.) Poit.) con $3(0.40 \%)$, belén (Impatiens balsamina L.) con $2(0.26 \%)$, campanilla o campanita (Ipomoea batatas (L.) Lam.) con $2(0.26 \%)$, batatilla (Ipomoea trifida (Kunth) G. Don.) con 3 (0.40), campanilla (Ipomoea triloba L.) con 3 (0.40), sangregrado o sangre de grado (Jatropha curcas L.) con $5(0.66 \%)$, nogal silvestre (Juglans major (Torr.) A. Heller) con 8 (1.06\%), Kallstroemia grandiflora Torr. ex A. Gray con $2(0.26 \%)$, alacate o bule (Lagenaria siceraria (Molina) Standl.) con $4(0.53 \%)$, mangle blanco (Laguncularia racemosa (L.) C. F. Gaertn.) con 20 (2.64\%), Guachichil (Loeselia mexicana (Lamb.) Brand) con 8 (1.06\%), hierba del golpe (Lopezia racemosa Cav.) con 8 (1.06\%), Ludwigia octovalvis (Jacq.) P.H. Raven con 27 (3.56\%), tepehuaje, palo fierro (Lysiloma acapulcense (Kunth) Benth.) con 2 (0.26\%), manzanito (Malpighia mexicana A. Juss.) con $5(0.66 \%)$, chilacayotillo o chilacayote (Melothria pendula L.) con $13(1.72 \%)$, lechosa (Mikania cordifolia (L.f.) Willd.) con 4 (0.53\%), cuatantillo o sierrilla (Mimosa albida Humb. y Bonpl. ex Willd.) con 6 (0.79\%), coatante (Mimosa pigra L.) con $3(0.40 \%)$, bálsamo (Momordica charantia L.) con 9 (1.19\%), tabaco (Nicotiana tabacum L.) con $3(0.40 \%)$, coquito de aceite (Orbignya guacuyule (Liebm. Ex Mart.) Hern.-Xol), palo de agua o zapote de agua o clavellina (Pachira aquatica Aubl.) con $2(0.26 \%)$, Paullinia sessiliflora Radlk con 3 (0.40\%), frijol (Phaseolus lunatus L.) con 8 (1.06\%), frijol blanco (Phaseolus vulgaris L.) con 12 (1.58\%), Phyla nodiflora (L.) Greene con 2 (0.26\%), guamúchil (Pithecellobium dulce (Roxb.) Benth.) con 7 (0.92\%), Guamuchilillo (Pithecellobium lanceolatum (Humb. y Bonpl. ex Willd.) Benth.) con $6(0.79 \%)$, alinanché o salvia de playa (Pluchea odorata (L.) Cass) con 2 (0.26\%), piojo (Porophyllum punctatum (Mill.) S.F. Blake) con 13 (1.72\%), verdolaga (Portulaca oleracea L.) con 2 (0.26\%), mamey (Pseudobombax ellipticum (Kunth) Dugand) con 7 (0.92\%), guayabo (Psidium guajava L.) con 7 (0.92\%), tapaojo o llamarada o mal de ojo (Psittacanthus calyculatus (DC.) G. Don) con 9 (1.19\%), Richardia scabra L. con $5(0.66 \%)$, higuerilla (Ricinus communis L.) con 4 (0.53\%), Salvia lasiocephala Hook. y Arn. con 8 (1.06\%), salvia morada (Salvia mexicana L.) con 18 (2.37\%), Sclerocarpus sessilifolius Greenm con $5(0.66 \%)$, chayotillo (Sicyos deppei G. Don.) con $8(1.06 \%)$, malva amarilla (Sida 
acuta Burm. f.) con 18 (2.37\%), huinar (Sida rhombifolia L.) con 23 (3.03\%), primavera (Tabebuia chrysantha (Jacq.) G. Nicholson) con 4 (0.53\%), amapa o rosa morada (Tabebuia rosea (Bertol.) A. DC.) con 4 (0.53\%), Tagetes erecta L. con 14 (1.85\%), almendro (Terminalia catappa L.) con $2(0.26 \%)$, alolote (Thevetia ovata (Cav.) A. DC.) con 9 (1.19\%), girasol (Tithonia tubiformis (Jacq.) Cass.) con $4(0.53 \%)$, asapescado (Trichilia hirta L.) con 6 (0.79\%), (Verbesina greenmanii Urb.) con 9 (1.19\%), Vernonanthura patens (Kunth) H. Rob. Con 10 (1.32\%), ahualamo (Vitex mollis Kunth) con 7 (0.9\%2), malva de monte (Waltheria indica L.) con $10(1.32 \%)$ y zinnia (Zinnia angustifolia Kunth) con 28 (3.69\%).

Las plantas con mayor número de lugares registrados, fueron: copalillo o corta pico (Bursera penicillata (DC.) Engl.) con 35 (4.62\%), guásima (Guazuma ulmifolia Lam.) con 28 (3.69\%), zinnia (Zinnia angustifolia Kunth) con 28 (3.69\%) y calavera (Ludwigia octovalvis (Jacq.) P.H. Raven con 27 (3.56\%).

Otras 95 especies con potencial apícola que se encuentran en Nayarit, son espino (Acacia berlandieri Benth.), huizache, huinol (Acacia farnesiana (L.) Willd.), cocoyul (Acrocomia mexicana Karw. ex Mart.), Agave americana L., marañón (Anacardium occidentale L.), aceitilla grande (Bidens pilosa L.), belladona (Atropa belladonna (L.), mostaza (Brassica nigra (L.) W.D.J. Koch), Bursera graveolens (Kunth) Triana y Planch, Cajanus cajan (L.) Huth, frijolillo (Calopogonium caeruleum (Benth.) C. Wright), palo blanco (Casearia sylvestris Sw.), zapote blanco (Casimiroa edulis La Llave y Lex.), ceiba, pochote (Ceiba aesculifolia (Kunth) Britten y Baker F.), ceiba (Ceiba pentandra (L.) Gaertn.), Citrullus vulgaris Schrad., alcachofa cimarrona (Cleome spinosa Jacq.), coco (Cocos nucifera L.), café (Coffea arabica L.), Coleus blumei Benth., Cordia alba (Jacq.) Roem. y Schult., amapa boba (Cordia alliodora (Ruiz y Pav.) Oken), huaché (Cordia globosa (Jacq.) Kunth), Croton fragilis Kunth, Cryptostegia grandiflora R. Br., Cuscuta americana L., Erythroxylum havanense Jacq., flor de nochebuena (Euphorbia pulcherrima Willd. ex Klotzsch), Funastrum clausum (Jacq.) Schltr., Genipa americana L. jagua, algodón (Gossypium hirsutum L.), lirio del arroyo (Hedychium coronarium J. Koenig), hibisco (Hibiscus rosa-sinensis L.), Ipomoea nil (L.) Roth, Lippia alba (Mill.) N.E. Br. ex Britton y P. Wilson, Lonicera japonica Thunb.ex Murray, Macfadyena unguis-cati (L.) A.H. Gentry, Baccharis trinervis Pers., bejuco loco (Cissus sicyoides L.), malva cubierta (Corchorus siliquosus L.), melón (Cucumis melo L.), mota (Dalea tomentosa (Cav.) Willd.), bejuco de jiote (Gouania polygama (Jacq.) Urb.), palo del Brasil (Haematoxylon brasiletto H. Karst.), Hamelia patens Jacq., salvia prieta (Hyptis rhytidea Benth), bastón de san Francisco (Leonotis nepetifolia (L.) R. Br.), guaje (Leucaena macrophylla Benth), monacillo (Malvaviscus arboreus Cav.), Melanthera nivea (L.) Small, paraíso (Melia azedarach L.), Merremia aegyptia (L.) Urb., albahaca (Ocimum basilicum L.), palo verde (Parkinsonia aculeata L.), guaco (Parthenocissus quinquefolia (L.) Planch.), aguacate (Persea americana Mill.), Piscidia piscipula (L.) Sarg, cruz esína (Pisonia aculeata L.), bejuco de huico (Pithecoctenium echinatum (Jacq.) Baill), llantén (Plantago major L.), chilillo (Polygonum punctatum Elliott), mareño (Prosopis juliflora (Sw.) DC.), caña (Saccharum officinarum L.), cola de pato (Sagittaria lancifolia L.), amole 
(Sapindus saponaria L.), bejuco cuadrado (Serjania triquetra Radlk), zacate de agua (Sesbania macrocarpa Muhl. ex Raf.), Sida abutifolia Mill., huinar chino (Sida spinosa L.), Sideroxylon salicifolium (L.) Lam., caoba (Swietenia macrophylla King), pastorcita (Tagetes patula L.), Talipariti tiliaceum (L.) Fryxell, verde lucero (Thouinia paucidentata Radlk), Thunbergia grandiflora Roxb, árnica (Tithonia diversifolia (Hemsl.) A. Gray), acahual (Tithonia rotundifolia (Mill.) S. F. Blake), Toxicodendron radicans (L.) Kuntze, Tradescantia zebrina Heynh, Tridax dubia Rose, Turbina corymbosa (L.) Raf., chamizo, andan chino (Viguiera dentata (Cav.) Spreng), Viguiera helianthoides Kunth y Vitex pyramidata B.L.Rob.

Muchas plantas son consideradas como malezas, como lo indica un estudio en Nayarit, donde registraron 127 especies como malezas (Hanan y Heike, 2015), del total de géneros de angiospermas presentes en Nayarit, que según Villaseñor (2003) son 1028 y según Téllez 968, de los que reportó 182 familias y 3650 especies (Téllez, 1995). Todavía más extremo, Villaseñor y Espinosa (1998) realizaron un listado de 821 especies consideradas maleza, las cuales cada día están en riesgo de eliminación.

Las plantas encontradas en este trabajo concuerdan con lo reportado por SAGARPA, que indica la presencia en esta región de plantas multifloral y de mangle (SAGARPA, 2018); ya que en Nayarit se encuentran once tipos de vegetación: los bosques tropicales subcaducifolio y caducifolio, el bosque mesófilo de montaña, los bosques de coníferas y de Quercus, el palmar, el manglar, la sabana de Byrsonima y Curatella, el bosque de galería, la vegetación acuática, la vegetación halófila y las asociaciones secundarias. La flora pertenece al reino neotropical, sin dejar de tener representación boreal; también se ubicó en la región Caribeña, que pertenece a la provincia de la Costa Pacífica (Téllez, 1995).

En un estudio realizado en la región de Pátzcuaro, Michoacan, México, se encontró que Apis mellifera visita y aprovecha 93 especies diferentes (Araujo-Mondragón y RedondaMartínez, 2019). En un estudio en las mieles producidas el estado de Baja California, se encontró la presencia de Tamarix spp. (pino salado), en el $100 \%$ de las mieles analizadas, seguido de Prosopis spp. (mezquite y tornillo), Medicago sativa (alfalfa), $P$. sericea (Cachanilla) y el tipo Chen-Am (Chamizos y quelites), presentes en el $92 \%, 90 \%$ y $87 \%$ de las muestras analizadas respectivamente. Otros tipos polínicos con alta frecuencia de ocurrencia, fueron: Myrtaceae (eucaliptos y cepillo rojo), en $81 \%$, seguido por W. filifera (palma de abanico), S. irio (mostacilla), Poaceae (pastos), y S. gooddingi (sauz), cada uno presente en $76 \%$ de las muestras analizadas. Otros hallados con valores bajos en las muestras, fueron: Gossypium hirsutum, Baccharis salicifolia, Acacia saligna, Cucumis melo, Malvella leprosa, Heterotheca, Heliotropium curassavicum, Opuntia , y Populus fremontii (Alaniz-Gutiérrez et al., 2017).

SAGARPA recomienda que los apicultores lleven a cabo trabajos de recuperación de la flora nativa con amplia variedad de arbustos, árboles y flores, en donde recolectar néctar y polen, tales como: acopio, conservación y reproducción de semillas; con la finalidad de 
diseminarlas para incrementar las plantas melíferas y por ende las cosechas, sobre todo si florecen en forma alterna (SAGARPA, 2018).

\section{CONCLUSIÓN}

El presente estudio reporta el primer censo de 177 especies de plantas, que son pecoreadas por abejas en el estado de Nayarit, México; de ellas, 82 plantas se cuenta con nombre científico, nombre común, fotografía y ubicación geográfica. También se describen 95 especies con potencial apícola, con el nombre común y científico; de acuerdo a la diversidad botánica de especies vegetales naturales o de producción artificial en el estado de Nayarit y de las cuales las abejas pecorean, puede ser mayor.

\section{Agradecimientos}

Este estudio fue financiado por Fundación Produce de Nayarit A. C. con folio 311-0439 a través del Sistema Producto Apícola de Nayarit. También se agradece por todo el apoyo en la identificación de las plantas a la Dra. Ana Hanan-Alipi de la Unidad Académica de Agricultura, Universidad Autónoma de Nayarit, México.

\section{Información Suplementaria.}

Archivo 1. Plantas con nombre científico, nombre común, fotografía y su geolocalización en mapas independientes.

\section{LITERATURA CITADA}

ALANIZ-Gutiérrez L, Ail-Catzim CE, Villanueva-Gutiérrez R, Delgadillo-Rodríguez J, Ortiz-Acosta ME, García-Moya E, Medina Cervantes TS. 2017. Caracterización palinológica de mieles del Valle de Mexicali, Baja California, México. Polibotánica. 43: 255-283. http://dx.doi.org/10.18387/polibotanica.43.12. http://www.scielo.org.mx/scielo.php?pid=S1405-

27682017000100255\&script=sci_arttext\&tIng=pt

ARAUJO-Mondragón F, Redonda-Martínez R. 2019. Flora melífera de la región centroeste del municipio de Pátzcuaro, Michoacán, México. Act. Bot. Mex. 126: e1444. http://dx.doi.org/10.21829/abm126.2019.1444

http://www.scielo.org.mx/scielo.php?script=sci_arttext\&pid=S0187-71512019000100130

ULLOA Castañeda RR, Anzaldo Velázquez JE, Martínez Vírgen M, Martínez González S, Loya Olguín JL. 2014. Generación de un modelo para la determinación de costos de empresas productoras de miel en el estado de Nayarit. Revista Mexicana de Agronegocios. 35:1072-1081. https://www.redalyc.org/pdf/141/14131676016.pdf. 
GARCÍA-Gómez LE y Meza-Ramos E. 2013. Oportunidades y obstáculos para el desarrollo de la apicultura en Nayarit. Universidad Autónoma de Nayarit. Pp.140. México. ISBN-13: 978-84-15774-25-9. https://www.eumed.net/libros-gratis/2013/1247/indice.htm

VARGAS-Valero A, Reyes-Carrillo J, Moreno-Reséndez A, Véliz-Deras F, GasparRamírez O, Rodríguez-Martínez R. 2020. Residuos de plaguicidas en miel y cera de colonias de abejas de La Comarca Lagunera. Abanico Veterinario. 10(1):1-16. http://dx.doi.org/10.21929/abavet2020.7

SAGARPA. Secretaría de Agricultura, Ganadería, Desarrollo Rural, Pesca y Alimentación. 2018. Manual de buenas prácticas pecuarias en la producción de miel. Pp. 79. México.

https://www.gob.mx/cms/uploads/attachment/file/395732/Manual_BPP_en_la_Producci _n_primaria_de_Miel_octubre_2018.pdf

HANAN Alipini AM, Heike Vibrans A. 2015. Las malezas: un laboratorio natural para el estudio de la evolución. Revista Fuente Nueva Época. 21:41-47.

https://www.researchgate.net/publication/295161239_Las_malezas_un_laboratorio_nat ural_para_el_estudio_de_la_evolucion

TÉLLEZ VO. 1995. Flora, Vegetación y Fitogeografía de Nayarit, México. Ciencias. 38:52-54. https://www.revistaciencias.unam.mx/es/191-revistas/revista-ciencias38/1805-flora,-vegetaci\%C3\%B3n-y-fitogeograf\%C3\%ADa-de-nayarit,m\%C3\%A9xico.html

VILLASEÑOR JL. 2003. Diversidad y distribución de las Magnoliophyta de México. Interciencia. 28(3):160-167.

http://ve.scielo.org/scielo.php?script=sci_abstractypid=S0378-

$18442003000300008 \mathrm{ylng}=$ esynrm=isoytlng=es

VILLASEÑOR RJL, Espinosa GFJ. 1998. Catálogo de malezas de México. Universidad Nacional Autónoma de México-Consejo Nacional Consultivo Fitosanitario-Fondo de Cultura Económica, México. 